\title{
EL CONCEPTO JURÍDICO DE LA REVOLUCIÓN
}

\author{
Ulises Schmill \\ Instituto Tecnológico Autónomo de México
}

RESUMEN. El objeto fundamental de este ensayo es presentar las herramientas conceptuales de carácter jurídico que permitan tener un concepto unitario tanto de los órdenes jurídicos avanzados en su centralización, como son los Estados modernos, como de un fenómeno históricamente muy reiterado y jurídicamente poco comprendido como son las revoluciones políticas. Tener un concepto unitario de los órdenes jurídicos avanzados y de las revoluciones significa tener la posibilidad de aplicar a los diversos fenómenos históricos mencionados un mismo conjunto de conceptos. Ello supone que disponemos de una teoría unitaria lo suficientemente poderosa para poder llevar a cabo la aplicación de la misma a los fenómenos históricos mencionados.

Palabras clave: teoría jurídica de las revoluciones políticas, orden revolucionario.

ABSTRACT. The aim of this paper is to present the conceptual legal tools which enable us to have a unitarian concept of both legal order with an advanced degree of centralisation, as is the case in modern states, and of the political revolutions, a historically very frequent and legally little understood phenomenon. Having a unitarian concept of advanced legal orders and of revolutions means being able to apply the same set of concepts to the afore mentioned historical phenomenon. This means that we have a unitarian theory powerful enough to be applied to the above historical phenomenon.

Keywords: legal theory of political revolutions, revolutionary order. 


\section{EXIGENCIAS PARA TODA TEORÍA JURÍDICA}

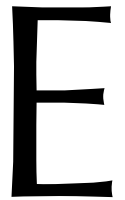

xiste una exigencia específica válida para cualquier teoría general del Derecho que se elabore, consistente en que pueda ser capaz de utilizarse para explicar, no solamente los problemas que tradicionalmente constituyen su objeto de estudio, sino nuevos problemas que surjan en ella o, por lo menos, un tratamiento mas sencillo, elegante o económico de esos problemas. Creo que es una decisión razonable preferir aquella teoría que resuelva de manera más sencilla y completa la problemática de la teoría jurídica. Puede ilustrarse este principio con dos ejemplos paradigmáticos: por razones puramente teóricas, es preferible la teoría del primado del orden jurídico internacional sobre el primado del orden jurídico nacional, porque aquélla maneja con mayor facilidad los problemas relativos a la estructura del orden jurídico y, además, resuelve más problemas de los que puede dar cuenta el primado del orden nacional, en especial, la permanencia e identidad del Estado nacional que sufre una revolución triunfante. El otro ejemplo es el de la identidad del Derecho y del Estado, es decir, el tratamiento jurídico de los problemas relativos a la esencia y estructura del Estado, frente a problemas sociológicos o de teoría económica sobre las actividades estatales, las que presuponen el concepto jurídico del Estado.

\section{2. ¿QUÉ ENTENDEMOS POR EXPLICACIÓN?}

Con base en este principio, podemos exigir que la teoría jurídica sea capaz de explicar al fenómeno de la revolución. Por explicación entiendo la posesión de aquellos conceptos necesarios para comprender el fenómeno revolucionario y poder identificar su existencia empíricamente. Ésta no es una tarea sencilla, pues se acostumbra pensar que la revolución, mientras dura la lucha revolucionaria, es un período en el que reina la anomía, contrariamente a la situación que prevalecía previamente en un Estado relativamente ordenado: se hace hincapié en los actos crueles de los revolucionarios, con el objeto de mostrar cuál es la naturaleza humana, como en Tucídides. Véase el siguiente ejemplo tomado de la Historia de la Guerra del Peloponeso:

Durante los siete días... los corcirenses se dedicaron a matar a los que consideraban enemigos personales suyos. Las acusaciones se dirigían contra los que intentaron derrocar el régimen democrático, pero también murieron algunos por causa de enemistades privadas y otros a manos de sus deudores. La muerte se mostró en mil formas diversas, y como sucede en circunstancias como ésta, no hubo exceso que dejara de suceder... y cosas peores aún. Los padres mataron a sus hijos, los suplicantes eran arrancados de los templos y matados en sus inmediaciones, e incluso algunos fueron rodeados de un muro en el templo de Dionisos, y murieron allí.

Tal fue la crueldad con que se desarrollaron las luchas civiles de Corcira y aún pareció mayor porque fue allí donde primero estallaron; pues más tarde toda Grecia, por así decirlo, fue desgarrada por las discordias que en todas partes había entre los jefes del partido popular, que querían llamar en su ayuda a los atenienses, y los aristócratas, que querían hacer lo propio con los lacedemonios... Muchos fueron los horrores que sufrieron las ciudades en las revoluciones, horrores que suceden y sucederán siempre mientras sea la misma la naturaleza humana, pero que son mayores o menores y de distinto carácter, según como sean las diversas circunstancias que se dan en cada ciudad... se hallaban pues en estado de revolu- 
ción las ciudades y las que tardaban más en entrar en él, al tener conocimiento de las cosas que ya habían sucedido, llevaban aún más lejos este cambio de conducta, tanto en lo referente al refinamiento de los que se lanzaban al ataque como en lo relativo a lo inaudito de las venganzas (Tucídides. HGP: 3.81.4 to 3.82.4).

Hay un profundo y, además, bellísimo párrafo en Tucídides que relaciona a la revolución con el uso del lenguaje:

Cambiaron incluso, para justificarse, el ordinario valor de las palabras. La audacia irreflexiva fue considerada valiente adhesión al partido, la vacilación prudente, cobardía disfrazada, la moderación, una manera de disimular la falta de hombría, y la inteligencia para todas las cosas, pereza para todas. Por el contrario, la violencia insensata fue tomada como algo necesario a un hombre, y el tomar precauciones contra los planes del enemigo, un bonito pretexto para zafarse del peligro. Los exaltados eran siempre considerados leales, y los que les hacían objeciones, sospechosos. Si uno urdía con éxito alguna maquinación, era inteligente, y más hábil aún si la descubría; en cambio, el que tomaba precauciones para que no le hiciera falta apelar a estos procedimientos, era considerado como traidor al partido y temeroso de los enemigos. En una palabra, el que se adelantaba a un enemigo que quería causarle un mal, era alabado, e igualmente el que invitaba a comportarse así a otro que no tenía esa intención. Y hasta tal punto fue esto así, que los lazos de la sangre llegaron a tener menos fuerza que los del partido, ya que éste estaba más dispuesto a mostrar una audacia sin miramientos; pues estas asociaciones no buscaban un beneficio público, guiándose de las leyes en vigencia, sino, violándolas, el abuso del poder. Las promesas de fidelidad recíproca no las confirmaban tanto con los ritos tradicionales como con la complicidad en el crimen (Tucídides. HGP: 3.82.4-6).

En estos párrafos clásicos del más grande de los historiadores encontramos una multiplicidad de problemas teóricos que ameritan su esclarecimiento, como lo intentó HobBES en su Leviatán. Todos estos enunciados sobre la revolución de Corcira se encuentran plenos de supuestos que hay que traer a la luz y que representan diversos puntos de vista desde los cuales se puede comprender el fenómeno de las revoluciones ( $c f r$. PRICE, 2001). Esto no implica que las causas de una revolución o sus efectos puedan determinarse jurídicamente, pues esa es la tarea de otras disciplinas distintas de la jurisprudencia, como la sociología, la historia, la economía, la antropología, etc. Tampoco estamos intentando explicar, con una teoría jurídica de las revoluciones, las justificaciones asociadas con todo movimiento revolucionario y las que lo justifican o legitiman. Tampoco intentamos vincular estos elementos de la ideología de los movimientos revolucionarios (en el sentido no peyorativo de la palabra «ideología») con cualquier concepción de mundo o de la vida (Weltanschauungen) de carácter filosófico, metafísico o teológico.

Una explicación jurídica de las revoluciones sólo significa presentar los conceptos normativos necesarios para identificar y reconocer la existencia de una revolución y del o de los movimientos revolucionarios que la preceden. Esto permitirá adicionalmente la aplicación de la teoría aceptada o postulada del orden jurídico a los fenómenos revolucionarios.

Nosotros nos preguntamos por la forma jurídica de comprender estos fenómenos y la razón de ello se encuentra en que las revoluciones son fenómenos políticos dirigidos a la destrucción de un Estado, lo que significa que están dirigidas a la sustitución de un orden jurídico por otro, en la medida que entendemos que el Derecho es un orden que regula el ejercicio del poder coactivo. 


\section{3. ÁMBITO FENOMÉNICO DE LA TEORÍA JURÍDICA TRADICIONAL}

Las explicaciones que se han construido para explicar jurídicamente las revoluciones se refieren generalmente a una revolución triunfante y poco o nada se dice en relación con el movimiento revolucionario, antes de su triunfo o fracaso. Un ejemplo de ello es la tesis de KELSEN sobre la revolución. KELSEN analiza el concepto de la revolución relacionándolo con el concepto de la norma fundante básica, pero sólo en el caso de que exista una revolución triunfante o fracasada. Considera lo que denomina principio de legitimidad, que expresa que la norma de un orden jurídico vale durante todo el tiempo que transcurra hasta que su validez no se extinga de una manera determinada por el propio orden jurídico (KelSEN, 1979: 217). Este principio, afirma, se encuentra limitado en el caso de una revolución, la que es concebida, en consecuencia, como cualquier modificación no legítima de la Constitución, es decir, efectuada de manera no conforme con las disposiciones constitucionales, o su reemplazo por otra. No importa si este reemplazo o modificación se hace con el uso de la fuerza o sin el uso de ella. Lo que importa es que la modificación o extinción de la constitución no se realiza conforme a sus prescripciones (KELSEN, 1979: 218). Estas afirmaciones son verdaderas si se cumple con la siguiente condición: el nuevo orden establecido, que sustituye al anterior, debe ser eficaz. Esto significa que sólo bajo la condición de la eficacia del sentido subjetivo de los actos de mandato es posible correctamente proyectar en los hechos el modelo que se haya construido de las normas y el orden jurídico y considerar que los actos de mandato que se hayan realizado constituyen normas válidas. Aquí existe un acercamiento, muy peligroso para el pensamiento de KELSEN, entre el concepto de validez y el de la eficacia. El «verse obligado a...» de HART se transforma en «estar obligado a...» (HART, 1968: 103) cuando el sentido de los actos de emisión de un mandato son eficaces, lo que permite la proyección sobre ellos del modelo de norma y de orden jurídico que se haya elaborado. Dice KELSEN:

Desde el momento que la antigua constitución ha perdido su eficacia, y la nueva la ha adquirido[...], los actos que aparecen con el sentido subjetivo de producir y aplicar normas jurídicas, no son interpretados ya presuponiendo la antigua norma fundante básica, sino la nueva (Kelsen, 1979: 219).

Un orden que pierde su eficacia es un orden cuyas normas han sido desconocidas, es decir, a las que se les ha negado el reconocimiento ( $c f r$. infra, apartado X). Este desconocimiento equivale a la invalidez de las normas que se inejecutan.

\section{NECESIDAD DE UNA TEORÍA JURÍDICA DE LAS REVOLUCIONES}

Todo lo anterior nos fuerza a hacer la siguiente pregunta: ¿qué significa hacer una teoría jurídica de la revolución? ¿Por qué es necesaria una teoría jurídica de la revolución? El ejemplo de KELSEN nos ilustra sobre ello. Ha planteado el problema de la revolución y ha dado una respuesta de carácter normativo. La tesis es inobjetable. Lo que no ha planteado ni, por tanto, ha tomado posición es sobre el siguiente problema: ¿cuál es la dimensión jurídica del movimiento revolucionario, mientras dura, antes de 
que se pueda considerar que ha triunfado o fracasado? Ya no nos preguntamos sobre el orden jurídico creado con la revolución triunfante o por el orden jurídico anterior, derogado por el nuevo, sino por el carácter jurídico de los actos integrantes del movimiento revolucionario, antes de que triunfe o fracase. Ciertamente, cuando un movimiento revolucionario ha triunfado el sentido subjetivo de los actos de mandato de los órganos del nuevo orden son interpretados conforme a una nueva Norma Fundante Básica, que determina cuáles son los órganos fundamentales, originarios, constituyentes, creadores del nuevo orden. Los órganos del orden anterior son considerados, dentro de la perspectiva del nuevo orden jurídico constituido teóricamente con la nueva Norma Fundante Básica, como delincuentes, sometidos al poder coactivo de los órganos del nuevo orden jurídico. La justificación de esta afirmación se encuentra en el hecho verificable de que los actos de mandato reales emitidos por los órganos del orden anterior ya no son interpretados con el punto de vista aportado por la nueva Norma Fundante Básica.

Surge en este lugar, entonces, el problema acuciante de cuál es el sentido y la cualidad de los actos integrantes del movimiento revolucionario. La primera reacción es negarles toda dimensión jurídica y verlos como actos de mera fuerza y violencia, si es que hay lucha armada, que es lo más frecuente, lo que no siempre es cierto, pues como tendremos oportunidad de demostrar, hay actos revolucionarios que no entrañan el uso de la violencia física , aunque sí dan origen a ella. Si se opta por esta primera alternativa la conciencia puede quedar en paz, pues con la teoría jurídica que poseemos podemos dar cuenta de fenómeno, negándole el carácter jurídico. La teoría queda incólume y nos podemos sentir satisfechos de poseer una teoría completa y satisfactoria, toda vez que el movimiento revolucionario no constituye un fenómeno histórico que deba ser explicado normativamente. No hay necesidad de revisarla ni inquirir sobre los presupuestos sobre los que descansa. Ellos pueden permanecer en el ámbito oscuro del silencio, en la esfera de lo no formulado. La segunda alternativa es reconocer el carácter jurídico de los actos integrantes del movimiento revolucionario, lo cual genera la necesidad de revisar los conceptos centrales de la Teoría General del Derecho para que puedan ser capaces de dar cuenta del carácter jurídico de los actos integrantes del movimiento revolucionario, mientras éste movimiento se desarrolla y extiende en el tiempo, sin haber llegado a la conclusión final de su triunfo o fracaso.

Una teoría jurídica de la revolución es necesaria por las siguientes razones:

1. La revolución es un fenómeno social esencialmente relacionado con el Estado, pues se dirige contra él con el propósito de destruirlo y sustituirlo por otro.

2. El criterio del acto estatal se encuentra en las normas jurídicas fundamentales. El acto estatal es acto jurídico de manera esencial. El criterio para determinar la cualidad de estatal de un cierto acto se encuentra en el contenido de las normas jurídicas que así lo establecen.

3. En consecuencia, la revolución debe tener una dimensión jurídica, pues con ella se deroga un orden jurídico y se instaura otro. Sólo jurídicamente pueden llevarse a cabo funciones jurídicas.

4. El ejemplo de Tucídides nos muestra que es posible hacer consideraciones de diversa índole sobre la revolución, pero la consideración primera y fundamental, en el sentido de estar presupuesta por las otras, es la jurídica. 


\section{5. ¿QUÉ SIGNIFICA ESTAR EN POSESIÓN DE UNA TEORÍA JURÍDICA?}

Si se ha de hacer una teoría jurídica de la revolución hemos de ser capaces de contestar la cuestión de qué significa estar en posesión de una teoría jurídica de la revolución o de cualquier otro fenómeno social.

Poseer una teoría jurídica significa construir un modelo de las normas y del orden normativo. La construcción de este modelo puede realizarse y, en general, se ha realizado desde distintos puntos de vista. Algunas veces se ha deducido de premisas religiosas o morales, de la observación de ciertos hechos, de la naturaleza humana, de doctrinas teológicas, etc.

Considero que un camino fructífero para construir una teoría del Derecho y tener, por tanto, un modelo del mismo, consiste en generalizar a la teoría del mandato, como lo han hecho parcialmente los iusnaturalistas racionalistas como GROCIO, PUFENDORF, TOMASIUS, la escuela analítica inglesa de BENTHAM y Austin, así como el positivismo de KELSEN y HART.

Se puede construir una teoría jurídica y obtener, con ello, el concepto del Derecho como un orden coactivo y dinámico de normas, a partir del modelo del mandato, tal como lo han hecho Austin, KELSEn y HaRT. Este concepto del Derecho se obtiene si se lleva a cabo sobre modelo del mandato un procedimiento consistente en introducir en la semántica de los enunciados descriptivos del mandato todos los elementos que se encuentran de hecho en la situación de su emisión, en el acto ilocucionario respectivo. Se trata de introducir en la semántica de los enunciados jurídicos todas las implicaturas al estilo de GRICE que se obtienen de la situación de emisión del mandato ( $c f r$. GRI$\mathrm{CE}, 1989$ ). Por ejemplo, la amenaza que respalda al mandato, en un inicio concebida como estando fuera del mandato, se introduce en la semántica del enunciado descriptivo del mandato, de modo que no sea ajena o exterior a él, sino que sea, en dicho enunciado descriptivo, uno de los contenidos dispuestos o prescritos por el mandato mismo. Si se lleva a cabo el procedimiento señalado obtenemos el modelo de una norma que contiene la prescripción de un acto de coacción para el caso de que se cumpla una cierta condición. Si p entonces debe ser Sanción x, es el esquema de los enunciados descriptivos del mandato. El «deber ser» en la anterior expresión no tiene ni puede tener otro significado que el de expresar que la relación entre el hecho p previsto en abstracto en el contenido del mandato está unido, dentro del enunciado descriptivo del contenido del mandato, con el contenido del mandato que faculta la imposición de la sanción x. Como contenidos de la semántica de los enunciados jurídicos, los hechos relacionados en él no son hechos que puedan ser explicados causalmente. Con ello adquiere sentido empírico la distinción y separación conceptual entre lo que ha sido llamado el «ser» y el «deber ser» (cfr. SCHMiLL, 1987a, 1987b).

Como no existen mandatos en la naturaleza, entonces es necesario concebir al mandato como un acto ilocucionario que podemos simbolizar de la siguiente manera: $<a_{i}$ | $\left.n_{i}\right\rangle^{1}$, donde $\ll<a_{i} \mid \gg$ está por el acto de emisión del mandato $\mathrm{y} \ll \mid n_{i}>\gg$ es el enunciado descriptivo de la norma coactiva emitida por el acto $<a_{i} \mid$, que siempre es un acto humano. De manera análoga al procedimiento constructivo de la interiorización semánti-

${ }^{1}$ Cfr. infra2 para conocer el significado del símbolo. 
ca de la amenaza concomitante al mandato en el enunciado descriptivo del contenido de las normas, si el acto de emisión de la norma se interioriza también en la semántica del enunciado descriptivo de la norma que establece dicho acto, se obtienen los enunciados descriptivos de las normas que otorgan poderes o competencias (facultades) a ciertas personas, que son los órganos del orden normativo en cuestión. Esta característica del Derecho de tener normas que establecen los actos de creación de otras normas, es lo que constituye al Derecho como un orden dinámico de normas, conforme a KELSEN y que son las normas secundarias en la teoría de HART.

Con lo anterior, obtenemos el esquema de un orden normativo, que tiene la siguiente forma simplificada:

$$
<a_{1}\left|n_{1}>-<a_{2}\right| n_{2}>-<a_{3}\left|n_{3}>\ldots<a_{r}\right| n_{r}>.
$$

A esta serie de elementos normativos podemos denominarla «cadena normativa». Un conjunto de cadenas normativas cuando sus primeros eslabones son comunes a las diversas cadenas constituye un orden normativo que adopta la estructura de un árbol.

Si se posee una teoría sobre el Derecho, en la forma que hemos expuesto brevemente y, por tanto, se tiene un modelo del orden normativo, es posible proyectarlo sobre los hechos históricos y comprender los acontecimientos respectivos como funciones jurídicas. Es entonces posible transitar del «verse obligado a...» al «estar obligado a...» de HART, tesis que se encuentra originalmente en KELSEN:

Si se quita a la «norma» o al «deber ser» todo sentido, no tiene entonces sentido alguno afirmar: esto está jurídicamente permitido, aquello jurídicamente prohibido, esto me pertenece, aquello a ti, X está autorizado a, Y obligado a, etc. En una palabra, han perdido su significado los miles de enunciados en que se manifiesta diariamente la vida jurídica. Pues es algo muy distinto que yo diga: A está jurídicamente obligado a pagar 1.000 a B, de que diga: existe cierta probabilidad de que A pague 1.000 a B. Y es algo completamente distinto que yo diga: esta conducta es —en sentido legal— un delito, y ha de castigarse - conforme a la ley_-, de que diga: quien ha hecho esto será con toda probabilidad castigado. El sentido inmanente con el que el legislador se dirige al órgano que aplica la ley, este órgano —en la sentencia judicial y en el acto administrativo — al súbdito, el súbdito a otro súbdito en el negocio jurídico, no es aprehendido con la enunciación acerca del probable curso de la conducta futura... No se le oculta (a la Teoría Pura del Derecho) que esa significación específicamente normativa de ciertos hechos designada como «derecho», es el resultado de una interpretación posible que se da ante un supuesto básico dado, pero no el resultado de una interpretación necesaria... (KELSEN, 1946: 63-65).

En caso de que no se tenga un modelo normativo del orden jurídico o se asuma la postura del anarquista, que en el fondo no es otra cosa que la ausencia del modelo normativo para interpretar los hechos históricos, entonces será imposible comprender jurídicamente los hechos que para el jurista son funciones normativas y estatales. La interpretación jurídica de ciertos hechos históricos es una posibilidad teórica que se presenta sólo en el caso de que se interpreten esos hechos con el esquema conceptual proporcionado por el modelo del Derecho que se haya construido. La teoría es el a prio$r i$ necesario para toda comprensión de cualquier elemento que podamos considerar como un hecho. Los hechos siempre están constituidos a partir de modelos o teorías. No hay hechos en sí mismos, carentes de teoría.

Permítaseme introducir dos signos para indicar que se han llevado a cabo las dos operaciones conceptuales mencionadas más arriba, consistentes en la introducción en 
la semántica del mandato tanto la amenaza coactiva como el acto de emisión del mandato. Estos símbolos son los siguientes:

$\left\langle a_{i} \mid n_{i}\right\rangle .\left\langle\mid n_{i}\right\rangle \gg$ es el sentido del acto de emisión del mandato y $\ll<a_{i} \mid »$ está por dicho acto ilocucionario. Colocamos a $\langle n\rangle$ entre los signos $|.$.$\rangle para indicar que del acto$ de emisión del mandato sólo se toma como foco de atención el sentido o la semántica del mismo, después de haber interiorizado en su enunciado descriptivo, en su semántica, la amenaza inherente al mandato. Estos signos «|..>» deben ser complementados por los correspondientes en el lado izquierdo de la barra, con el objeto de obtener el concepto completo de toda norma jurídica positiva, que sólo puede considerase como existente si ha sido puesta o producida por un acto humano, cuyo sentido es: $\left\langle a_{i} \mid n_{i}\right\rangle$. $\mathrm{La} \ll\left|n_{i}\right\rangle \gg$ señala al concepto de la coactividad como contenido de la norma y $\ll<a_{i} \mid »$ señala al concepto de la positividad o dinamicidad del Derecho, en tanto se ha introducido en la semántica de la norma el contenido que indica el acto creador de otras normas. En realidad, podemos considerar que los signos $\langle<.|. » \mathrm{y}\langle\mid ..\rangle \gg$ significan que hemos realizado las operaciones conceptuales en los enunciados descriptivos del mandato, consistentes en introducir dentro de su semántica tanto la coacción como el acto de emisión del mandato.

Para construir un modelo jurídico de la revolución es necesario dar un paso consistente en establecer cuáles son las características específicas de los órdenes jurídicos avanzados y evolucionados, con el objeto de hacer sobre dicho modelo diversas operaciones y transformaciones a efecto de encontrar las notas definitorias de otros modelos de órdenes normativos. Esto lo haremos a continuación con el modelo del orden coactivo y dinámico de normas.

\section{DEFINICIONES FUNDAMENTALES}

Partimos del principio de que el objeto de estudio de la ciencia del Derecho o jurisprudencia son las normas de un orden jurídico positivo. La función fundamental de la jurisprudencia es la descripción, lo más precisa posible, de la estructura y modus operandi de un orden jurídico. Esto implica de manera obvia el rechazo de cualquier teoría cuyo objeto sea el estudio de normas no positivas, es decir, normas no creadas históricamente por ciertos actos humanos específicos.

Estos supuestos permiten afirmar que las normas que pertenecen a un orden jurídico positivo son descritas en proposiciones que han incorporado en su semántica todos los elementos presentes en la situación pragmática de la emisión de un mandato, incluyendo especialmente el mal con que se amenaza al recipiente del mandato en caso de incumplimiento. De esta manera concebimos al Derecho como un orden coactivo de la conducta humana; en otras palabras, como un orden que prescribe el ejercicio de la coacción cuando la conducta ordenada es omitida por el destinatario. No es el objeto de este ensayo elaborar el procedimiento intelectual por el cual en los enunciados descriptivos de las normas jurídicas se interiorizan los elementos presentes en la situación de emisión del mandato coactivo (cfr. SCHMILL, 1997). Lo que importa destacar es que las normas son el sentido de ciertos actos determinables empíricamente y que un orden jurídico positivo está constituido de una pluralidad de normas positivas con una estructura determinada, que más adelante se explicitará. 
A continuación presentaremos algunos conceptos fundamentales, que serán de gran utilidad en lo que sigue.

En primer lugar, un acto normativo es un acto cuyo significado es una norma, es decir, un acto cuya dimensión material o sustantiva de realización es una norma. El enunciado que las describe ha incorporado en su semántica, en la consecuencia del enunciado condicional respectivo, el mal con el que se amenaza al destinatario. La metáfora de la creación o producción de la norma puede usarse sin cometer el error de considerar a la norma como un producto distinto e independiente del acto. La norma es solamente el sentido o significado del acto ilocucionario de emisión del mandato: a(n). Sin embargo, para la finalidad del análisis teórico, es posible abstraer el sentido normativo del acto normativo, considerando al primero como la norma misma y referirse a ella de manera independiente.

Un orden jurídico positivo contiene una pluralidad de normas relacionadas entre sí de varias maneras, lo que nos permite concebirlas como un orden con una estructura interna dada. La imagen intuitiva más clara es la de una estructura arborescente en la que cada rama contiene una secuencia ordenada de una pluralidad de actos normativos. Una secuencia de esta índole puede ser representada con la imagen de una cadena normativa construida con una secuencia de actos normativos algunos de los cuales - los actos precedentes o determinantes - condicionan la realización de los actos subsecuentes o determinados, en tanto que el contendido de aquéllos son los actos de creación y el contenido de los actos subsecuentes. Podemos representar una cadena normativa de la siguiente manera:

$$
<a_{1}\left|n_{1}>-<a_{2}\right| n_{2}>-<a_{3}\left|n_{3}>\ldots<a_{r}\right| n_{r}>.
$$

El concepto de una cadena normativa posibilita un entendimiento mejor de lo que realmente es una norma precedente o determinante. Ésta es la norma que establece la facultad, cuyo contenido es la determinación de los actos o procedimientos y el contenido de otra u otras normas. Por ejemplo, $\mid n_{1}>$ con respecto a $<a_{2} \mid n_{2}>$; en general, | $n_{r-1}>$ con respecto a $\left\langle a_{r} \mid n_{r}\right\rangle$.

Consecuentemente, una norma subsecuente o determinada es la norma creada por ciertos actos o procedimientos cuyo contenido se encuentra determinado en las normas precedentes o determinantes; $\left\langle a_{2} \mid n_{2}\right\rangle$ con respecto a $\left|n_{1}\right\rangle$; en general $\left\langle a_{r} \mid n_{r}\right\rangle$ con respecto a $\mid n_{r-1}>$. Dada una cadena normativa, es posible determinar la longitud de la cadena normativa tomando en cuenta el número de elementos incluida en ella, es decir, los elementos que corresponden a las normas positivas en ella incluidas.

Si se acepta el esquema simple de una cadena normativa, entonces se pueden definir varios conceptos que son predicados que pueden hacerse de los actos normativos y las normas correspondientes, de la siguiente manera:

1. La existencia de una norma se establece siempre que sea posible determinar la relación entre el acto y la norma creada, entendida como la dimensión material o sustancial de realización de dicho acto; en otras palabras, siempre que pueda mostrarse que la norma es el sentido o significado de tal acto. Bajo esta definición, sería necesario afirmar la inexistencia de las normas del derecho natural. Se sostiene que esas normas no son el sentido de actos humanos específicos, sino de actos realizados por seres sobrehumanos o derivados de situaciones naturales. Con relación a este concepto de la 
existencia de una norma, si la relación es contemplada desde la perspectiva de la norma, se puede afirmar su positividad si es el sentido de un acto normativo. Esto lo podemos representar de la siguiente manera, tomando en cuenta el esquema de una cadena normativa. Como la existencia es una relación específica entre elementos de la cadena normativa, debemos seleccionar esos elementos en cada concepto y relacionarlos entre sí. Con la existencia tenemos: $\left[\mathrm{Ex}=\mathrm{R}_{\mathrm{ex}}\left(a_{\imath}, n_{i}\right)\right.$; ej. $\left.\mathrm{R}_{\mathrm{ex}}\left(a_{1}, n_{1}\right)\right]$. La positividad es la relación inversa de la existencia, por lo que es representada así: $\left[\operatorname{Pos}=\mathrm{R}_{\mathrm{pos}}\left(n_{z} a_{i}\right)\right.$; ej. $\mathrm{R}_{\mathrm{pos}}$ $\left.\left(n_{1}, a_{1}\right)\right]$.

2. Es conveniente concebir la validez de una norma como la relación entre la norma positiva condicionante y el acto que la aplica en la creación de otra norma [Val = $\left.\mathrm{R}_{\mathrm{val}}\left(n_{i}, a_{i}+1_{) ; \text {e e. } \mathrm{R}} \mathrm{val}_{(n 1}, a_{2}\right)\right]$. La relación inversa de la validez es el reconocimiento de una norma, dado que el acto normativo que la ejecuta necesariamente implica su reconocimiento. $\left[\operatorname{Rec}=\mathrm{R}_{\mathrm{rec}}\left(a_{i}+1,{ }_{n}\right) ;\right.$; ej. $\left.\mathrm{R}_{\mathrm{rec}}\left(a_{2}, n_{1}\right)\right]$ (cfr. KELSEN, 1996).

3. La legitimidad de una norma puede afirmarse si es posible determinar la relación entre la norma positiva condicionante y la norma creada o producida con el acto que la ejecuta [Leg $=\mathrm{R}_{\operatorname{leg}}\left(n_{z} a_{i}+1_{(n} i+1_{) \text {; ej. } \mathrm{R}} \operatorname{leg}_{(n 1}, a_{2}\left(n_{2}\right)\right]$. Si esta relación es contemplada desde la perspectiva de la norma positiva condicionada estamos en presencia del concepto de regularidad $\left[\mathrm{Reg}=\mathrm{R}_{\mathrm{reg}}\left(a_{i}+1_{(n} i+1_{) \text {, ni ej. } \mathrm{R}} \mathrm{reg}{ }_{(a 2}\left(n_{2}\right), n_{1}\right]\right.$.

Consecuentemente, el orden jurídico se presenta como un árbol cuyas ramas están constituidas por cadenas de actos normativos; esto es, de actos de mandato, de actos cuyos sentidos son las normas y que, cada uno, determina los actos normativos subsecuentes. Un orden normativo se forma por la operación recurrente del concepto de la validez de una norma previamente existente. Si una norma existe porque es el sentido o significado de un acto normativo, entonces su validez puede ser afirmada siempre que pueda mostrarse que es el fundamento de un acto normativo subsecuente.

\section{EL SIGNIFICADO DE LA DERROTABILIDAD}

Intentaremos ahora exponer cuál es el sentido del orden jurídico, es decir, cuál es la situación en que se encuentra un sujeto cuando está frente a una cadena de actos normativos, cuyo contenido es exigirle una serie de conductas determinadas. Para explicitar este problema debemos incursionar previamente en un concepto que ha sido desarrollado a últimas fechas y que es el concepto de la «derrotabilidad» de los enunciados normativos.

Primeramente debemos decir que por medio del concepto de la derrotabilidad de un enunciado condicional puede llegarse a expresar el sentido general de un orden normativo y representar la manera como se construye en los hechos una cadena normativa. Esto permitirá construir el concepto normativo de revolución.

Para ello, es conveniente aceptar la tesis de AlCHOURRÓn y BulYgin ( $c f r$. AlCHOURRÓN y BULYGIN, 1974) sobre la estructura de los enunciados que describen el contenido de las normas jurídicas. De manera similar a KELSEN, consideran que el contenido de cualquier norma puede ser expresado por medio de un enunciado condicional (KELSEN diría por medio de un «juicio hipotético»), en cuyo antecedente existe el enunciado que describe un supuesto determinado, simple o complejo, y en el consecuente una 
expresión que describe una cierta conducta modalizada deónticamente, es decir, como $\mathrm{P}$ (permitida), $\mathrm{Ph}$ (prohibida), O (obligatoria) y F (facultada). Puede denominarse enunciado condicional normativo al que tiene la forma condicional descrita, es decir, $\left|p \rightarrow X^{*} q\right\rangle$, en donde $\left\langle X^{*} »\right.$ está por los operadores deónticos mencionados. Estos enunciados describen el contenido de las normas correlacionando circunstancias específicas, generales o particulares, con soluciones normativas.

Debemos ahora exponer el concepto de la derrotabilidad de un enunciado condicional. Una caracterización muy clara de esta clase de enunciados la encontramos en VON WRIGHT, quien se expresa en los siguientes términos:

Si por sí misma implica q, entonces la conjunción del antecedente ( $\langle p »)$ con cualquier proposición diferente de él también implica $q$. La operación de pasar de «si $p$ entonces q» a «si $p \& r$ entonces $q »$ es denominada la operación del refuerzo del antecedente.

Si $p$ por sí misma no implica $q$, el condicional que dice que si $p$ entonces $q$ se denomina derrotable. Un condicional derrotable no permite (de manera irrestricta) la operación de refuerzo del antecedente (VON WRIGHT, 1966: 23).

El profesor Carlos AlCHOURRÓn investigó el problema lógico de los enunciados condicionales derrotables, es decir, de aquellos enunciados condicionales cuyo antecedente es una condición necesaria, pero no una condición suficiente, del consecuente del condicional. Dice:

En el lenguaje corriente las construcciones condicionales de la forma «Si A entonces B» son frecuentemente usadas de un modo tal que no se pretende con ellas afirmar que el antecedente A es una condición suficiente del consecuente B, sino sólo que el antecedente, sumado a un conjunto de presupuestos aceptados en el contexto de emisión del condicional, es condición suficiente del consecuente B. Este es el caso, por ejemplo, cuando se afirma, con relación a una cierta muestra de gas, que su volumen aumentará si se eleva la temperatura, suponiendo en el contexto que la presión se mantendrá constante. La afirmación condicional es derrotada cuando alguno de los presupuestos implícitos es falso. Un condicional derrotable también puede ser definido como un condicional sujeto a excepciones implíci$\operatorname{tas}^{2}$.

Si partimos del enunciado condicional normativo que describe una norma, la expansión del antecedente $p$ consiste en la adición, por medio de una conjunción, de los enunciados que describen los actos de creación y los contenidos establecidos en las normas precedentes en una cadena normativa determinada. El enunciado condicional descriptivo de la norma, con la expansión completa del antecedente $p$, contiene los enunciados descriptivos de los actos de creación y de los contenidos determinados por las normas precedentes y tiene la siguiente forma: $\mid p \cdot\left(a_{i} \cdot z_{j}\right) \rightarrow X^{*} q>$. Un enunciado que contiene la expansión de $p$ con los actos del procedimiento establecidos en las normas precedentes se denomina expansión adjetiva de p; los que contiene los contenidos que determinan las normas precedentes se denomina expansión sustantiva de $p$.

En realidad, los contenidos de las normas precedentes de una cadena normativa se encuentran, por así decirlo, proyectados en el antecedente del condicional normativo descriptivo del contenido de una norma subsecuente. Las normas precedentes contienen los criterios de regularidad e irregularidad, i.e., los criterios que permiten la cons-

${ }^{2}$ Alchourrón, 2000. Cfr. igualmente Navarro, 2000. Navarro y Rodríguez, 2000. Rodríguez y Sucar, 1998. 
trucción de los condicionales derrotables relativos a las normas subsecuentes. Los elementos adjetivos y sustantivos que pueden derrotar el enunciado descriptivo de una norma, no se presentan normalmente de manera explícita en los enunciados que describen una norma jurídica. La descripción de los contenidos de las normas precedentes permiten establecer los criterios de derrotabilidad de los enunciados descriptivos de las normas subsecuentes. Por lo tanto, las normas precedentes llevan a cabo dos funciones diferentes:

1. Función reguladora: la norma precedente establece los actos creadores de las normas subsecuentes, los cuales tienen especificadas sus dimensiones de realización, de entre las cuales es de especial importancia la dimensión material, pues ella contiene el sentido jurídico de los actos.

2. Función de criterio de la regularidad: las normas precedentes contienen los criterios con base en los cuales se pueden determinar de manera positiva las irregularidades en la realización de esos actos normativos constitutivos del proceso de creación de la o las normas subsiguientes.

Si se determina de manera positiva la existencia de una irregularidad adjetiva o sustantiva del acto de creación de una norma subsecuente, esto se expresa en el enunciado condicional normativo descriptor de la norma por medio de la adición de los enunciados que contiene la negación de una de las expansiones de $p$, i.e., $<a \mid p \cdot\left(\sim a_{i} v \sim z_{i}\right)$ $\rightarrow X^{*} q>$, lo cual produce la negación del elemento consecuente del condicional, i.e., la negación de la solución normativa, lo que significa la negación de la modalización deóntica de la conducta descrita en el consecuente del condicional normativo: $\left\langle a \mid \sim X^{*} q\right\rangle$. Esto significa que la conducta descrita por $q$ en el consecuente del enunciado condicional normativo, no se encuentra dentro del ámbito del operador deóntico, lo que sólo puede acontecer si ha existido un acto positivo que haya determinado la nulidad de la norma que establece esa conducta sujeta a un operador deóntico. Esta nulidad significa que hay un acto positivo de un órgano jurídico que ha determinado la fecha final de validez del ámbito temporal de la norma en cuestión, condicionada al establecimiento de alguna irregularidad adjetiva o sustantiva. Por lo tanto, todo enunciado descriptor de una norma por medio de un enunciado condicional canónico contiene una cláusula implícita de derrotabilidad. La «regularidad» de un acto o de una norma significa que el órgano encargado de aplicar una norma precedente ha establecido que se ha cumplido, en el acto de aplicación, con las expansiones de $p$.

\section{EL SUPUESTO FUNDANTE BÁSICO}

Nuestro concepto de «cadena normativa» se encuentra cargado de supuestos que deben esclarecerse. La afirmación de que una norma existe presupone, por lo menos, el concepto del acto de emisión del mandato y su dimensión material de realización, que es el sentido normativo del acto, es decir, presupone un modelo del acto normativo. Ello significa que se ha podido construir un modelo del acto ilocucionario del mandato, lo que no se hará en este lugar.

En la teoría de KELSEN, la norma fundante básica es un presupuesto lógico-trascendental. Por lo tanto, como presupuesto trascendental, no es ni puede ser una norma, 
como pareciera que piensa KELSEN, por el nombre que le atribuye «norma fundante básica» (norma fundamental hipotética), pues sería, entonces, en tanto norma, objeto de explicación y no la hipótesis explicativa. Por ello, es mejor concebir este presupuesto como el supuesto fundante básico.

Esto significa que se está en posesión de un modelo de orden normativo, el cual se ba proyectado sobre ciertos actos efectivamente realizados, para entenderlos y exponer sus funciones con base en los conceptos normativos obtenidos del modelo teórico utilizado.

Afirmar la existencia de una norma desde el punto de vista de la teoría del Derecho no es otra cosa que la proyección, sobre ciertos actos específicos de la experiencia, del modelo de la norma positiva construido teóricamente. Comprender algo como una norma existente presupone que se da en los hechos la relación definida por el concepto de existencia de una norma.

Entender algo como una norma existente presupone una situación donde la relación definida por el concepto de norma existente tiene lugar de hecho. Afirmar la existencia de una norma desde el punto de vista de una teoría del Derecho no es otra cosa sino la proyección — a ciertos actos de la experiencia- del modelo de las normas positivas construido teóricamente. Sin embargo, el concepto de la «validez» presupone el condicionamiento de un cierto acto normativo y su sentido de acuerdo con una norma determinante o precedente. El concepto de «validez» permite la construcción del concepto de «orden normativo», así como de otros conceptos distintos que más tarde se discutirán.

\section{EL SENTIDO DEL ORDEN NORMATIVO}

La anatomía del hombre es la clave para entender la anatomía del simio, dijo en algún lugar Carlos MARX. Creo que tiene razón. Tomando este consejo en serio, es conveniente precisar las características de un orden jurídico desarrollado, plenamente evolucionado, moderno y complejo, para con ello poder entender mejor el modus operandi tanto de los órdenes normativos primitivos como de las destrucciones de los órdenes desarrollados y, con ello, la revolución.

En un orden estático de normas, en el sentido definido por KeLSEN ( $c f r$ KelSEN, 1946: 94 y ss; 1979: 201 y ss; 1934: 325 y ss.) en el que las normas dependientes se deducen de la norma primera o fundamental, no se presenta el concepto de derrotabilidad, por el propio concepto constitutivo de esta clase de órdenes, pues están excluidas las contradicciones entre normas. Si una norma es deducida, la deducción garantiza su congruencia con la norma que le ha servido de premisa. La verdad o validez de las premisas se hereda a las conclusiones.

En cambio, en un orden positivo, en un orden dinámico en el sentido de KELSEN ${ }^{3}$, que establece procedimientos y órganos facultados para crear normas subsecuentes, las contradicciones hacen su aparición como posibilidad que no puede ser excluida, debido a que el órgano creador de la norma subsecuente debe dotarla de un contenido determinado siguiendo un procedimiento específico, los cuales pueden no concordar con

\footnotetext{
${ }^{3}$ Cfr. nota anterior.
} 
el contenido de la o las normas determinantes o precedentes. La contradicción entre las normas subsecuentes y las normas precedentes o determinantes son los elementos que derrotan al enunciado condicional normativo.

La tendencia hacia la unidad es el criterio fundamental para establecer el sentido del orden normativo, junto con el de poseer un conjunto de métodos o procedimientos para restablecerla, en caso de rompimiento de la misma. Esto puede exponerse, con base en el concepto de regularidad, de la siguiente manera: si un órgano jurídico (particular u oficial) se encuentra frente a una cadena normativa, el sentido del orden jurídico puede expresarse diciendo que debe obedecerse la norma individual que exige obediencia, si ha sido creada o emitida por el órgano competente y con el contenido autorizado por las normas precedentes; esa norma individual ha de ejecutarse porque tiene su fundamento en otra norma previa de la cadena normativa, por ejemplo, un reglamento y éste, además, fue emitido conforme a una ley expedida por el órgano legislativo; esta ley ha de ser ejecutada porque ha sido dictada por el Parlamento o el Congreso a través del proceso legislativo establecido en la Constitución y sin contravenir su contenido; la Constitución ha de ejecutarse porque ha sido emitida por el acto constituyente o por la costumbre. Este es el supuesto último de toda comprensión del material normativo.

Con los conceptos anteriores, podemos decir que el último y propio sentido del orden jurídico es determinar, a través de las normas generales precedentes en una cadena normativa, el procedimiento y los contenidos de los actos normativos individuales de los órganos jurídicos. Cuando se utiliza un enunciado condicional normativo para describir el contenido de una norma individual que exige cumplimiento, debe observarse que el contenido de las normas generales precedentes se encuentran consignadas dentro de la expansión del antecedente del enunciado condicional. Estos elementos de la expansión del antecedente del enunciado condicional, son los que pueden derrotar al condicional para el caso de que alguno o todos sean falsos, es decir, cuando signifique que no se han cumplido de manera regular. Estas afirmaciones están basadas en la experiencia ordinaria de los juristas de examinar cualquier norma en relación con las normas precedentes que determinan su forma de creación y su contenido.

La sola emisión de una norma por un órgano específico siguiendo un procedimiento determinado y con cierto contenido, no permite afirmar su regularidad. La regularidad de un acto normativo no es algo dado a priori que pueda determinarse desde el momento en que es emitida, sino que es la materia de una decisión específica de carácter positivo, ya sea por el órgano ejecutor de la norma, como lo pretenden KELSEN y HART, o por otro órgano encargado de revisar y decidir sobre su regularidad. Si un órgano determinado ejecuta una norma precedente, por la realización de este mismo acto está reconociendo a la norma que le sirve de fundamento y por ello puede predicarse la validez de la misma. Debemos relacionar estos fenómenos con nuestro concepto de reconocimiento, que es la relación existente entre el acto creador de una norma subsecuente y la norma determinante o precedente que le sirve de fundamento; es, como dijimos, el concepto inverso del concepto de validez. Una norma lleva a cabo su función específica, es válida por tanto, en el caso de que condicione de hecho la conducta, de manera que ésta se ajuste al sentido de la norma. Esto sólo es posible si el sujeto que aplica la norma la reconoce como tal, es decir, si decide que es una norma que exige cumplimiento y lleva a cabo la conducta que aquella especifica. 
En consecuencia, en presencia de una multiplicidad de cadenas normativas de longitud máxima puede predicarse la existencia de un orden jurídico. El sentido del orden jurídico consiste en la determinación de los actos y de los contenidos de las normas subsecuentes por los contenidos de las normas precedentes. Estas constituyen los parámetros para medir fundamentalmente la legitimidad y la regularidad de las normas que integran un orden jurídico.

\section{EL ORDEN JURÍDICO EVOLUCIONADO}

Si aceptamos la formulación del orden jurídico moderno avanzado que han hecho KELSEN, de manera muy completa, y HART, de manera relativamente incompleta, podemos decir que en un orden jurídico evolucionado encontramos las siguientes características o notas observables:

1. Es un orden coactivo de la conducta humana. Muchos protestarán por esta formulación escueta, pero en realidad en ella se encuentran los elementos esenciales de una concepción del Derecho más o menos completa. En primer lugar debemos observar que los conceptos centrales de esta concepción están expresados con dos palabras: es un «orden», lo que significa que es un conjunto de normas y es «coactivo», lo que significa que ese conjunto de normas tiene las notas definitorias del concepto kelseniano del orden dinámico de normas. Para aquellos que gustan de la teoría de HART tendremos que decir que el Derecho comprende tanto normas primarias (coactivas) como secundarias, pues es claro que la coacción no se genera de manera causal, espontánea, por sí misma, sino que tiene que ser establecida por seres humanos en contra de otros seres humanos cumpliendo con las normas que los facultan para ello. Por lo tanto, tenemos el concepto de un orden coactivo y dinámico de normas.

2. En principio, los ámbitos de validez de un orden jurídico avanzado se encuentran claramente delimitados y establecidos, por el orden mismo en concordancia con las normas del Derecho Internacional a través del reconocimiento de la existencia de dicho orden jurídico por los órganos de la orden jurídico internacional.

3. Por lo tanto, puede considerarse que el orden jurídico avanzado tiene fijados sus ámbitos de validez de manera permanente y rígida. Es lo que puede llamarse la rigidez de los ámbitos de validez del orden estatal.

4. Las cadenas normativas constitutivas del orden jurídico avanzado tienen una longitud máxima.

Estos son los conceptos más importantes que podrán ser utilizados para compararlos con los que se utilizarán en la determinación de las características de los órdenes revolucionarios.

Un par de conceptos adicionales nos serán de gran utilidad. Si hacemos objeto de nuestras consideraciones a un orden jurídico positivo moderno y evolucionado, al que podemos denominar orden primario evolucionado, podemos comprobar que el mismo tiene la característica de que las cadenas normativas que lo componen tienen la máxima longitud, en virtud de que los elementos que las integran son más numerosos que en otros órdenes no tan evolucionados. Por ejemplo, una resolución administrativa de la Secretaría de Hacienda que determina que un cierto individuo debe pagar, por con- 
cepto de impuesto sobre la renta, 100.000 pesos, puede ser expresada de la siguiente manera:

$$
\begin{aligned}
& n_{0}=<a_{0 \text { (acto jurídico) }} \mid n_{0 \text { (resolución fiscal) }}=p \text { (ingreso gravable) }\left[\cdot n_{1 \text { (Const) }}\left(a_{1} v z_{1}\right) .\right. \\
& \left.n_{2 \text { (ley ISR) }}\left(a_{2} v z_{2}\right) \cdot n_{3 \text { (Regl ISR) }}\left(a_{3} v z_{3}\right)\right] \rightarrow \mathrm{O} q_{\text {(obligación de pago de 100.000) }}>
\end{aligned}
$$

Puede apreciarse de manera inmediata que $n_{0}$ es la norma constitutiva de la resolución administrativa emitida por la Secretaría de Hacienda que la norma $n_{0}$ es la resolución administrativa dictada por la Secretaría de Hacienda, que es el objeto de las presentes consideraciones, y cuyo significado es que un determinado individuo ha obtenido ingresos gravables de cierta cuantía determinada y, consecuentemente, establece la obligación de pagar 100.000 pesos por concepto del impuesto sobre la renta, i.e., $\mid p \rightarrow$ $\mathrm{O} q>$. El esquema muestra la expansión de $p$, con los requerimientos adjetivos y sustantivos de las normas precedentes determinantes: la Constitución, la ley, el reglamento respectivo, indicando cómo las normas precedentes en un orden jurídico están proyectadas en la expansión del antecedente en el condicional derrotable, es decir, $\left[\cdot n_{1 \text { (Const) }}\right.$ $\left.\left(a_{1} v z_{1}\right) \cdot n_{2(\text { ley ISR) }}\left(a_{2} v z_{2}\right) \cdot n_{3 \text { (Regl ISR) }}\left(a_{3} v z_{3}\right)\right]$. Debe notarse que el enunciado condicional con la expansión del antecedente posee lo que he denominado «longitud máxima».

Por tanto, un orden normativo reducido será aquel cuyas cadenas normativas no contienen el primero, o los primeros, elementos de las cadenas normativas de un orden evolucionado. El fenómeno de la reducción de un orden normativo podemos considerarlo como el proceso real, que la jurisprudencia tiene que comprobar, por el que existen empíricamente uno o varios órdenes normativos reducidos. La reducción de un orden normativo entraña la descentralización de alguno de sus ámbitos de validez, debido a la generación de diversos órdenes normativos independientes. La unidad del orden normativo se encuentra garantizada por la existencia del Derecho Internacional, con su principio de efectividad.

Con las bases anteriores, el sentido del orden jurídico puede expresarse diciendo que, en la formulación de cualquier norma con un enunciado condicional derrotable, la expansión de $p$, contiene elementos, tanto adjetivos como sustantivos, que son el contenido de normas positivas precedentes. Los elementos que constituyen la expansión de $p$ pueden ser más o menos numerosos. De manera general, en un orden moderno evolucionado, estos elementos tienen una longitud igual a la longitud de la cadena normativa correspondiente, menos uno, para excluir la norma que se expresa en el condicional canónico. Las normas precedentes aparecen proyectadas como elementos que derrotan un enunciado condicional normativo.

\section{CONCEPTO DE REVOLUCIÓN Y ORDEN REVOLUCIONARIO}

Si se parte de un orden primario evolucionado, en el sentido definido anteriormente, como paradigma para comparar la existencia de cadenas normativas empíricas, entonces podemos observar que si no es posible afirmar la derrotabilidad de los enunciados condicionales correspondientes a ciertas normas, por la no inclusión en la expansión de $\mathrm{p}$, de los enunciados que describen los contenidos de las normas iniciales de un orden primario evolucionado, es debido a que se ba instaurado un orden revolucionario. 
Si $n_{0}=\mid p \rightarrow \mathrm{O} q>$ puede ser descrito totalmente con el esquema debido a la no existencia de la expansión del antecedente $p$ con el contenido de las normas precedentes, el enunciado mencionado significa que no hay normas precedentes determinantes del acto de creación y del contenido de $n_{0}$, en tanto no se encuentran contenidas en la expansión del antecedente. Esto sólo puede ocurrir en dos casos:

a) Del acto constituyente de un orden jurídico avanzado (si prescindimos momentáneamente del Derecho Internacional).

b) De un acto revolucionario.

Esto significa que las normas precedentes del orden primario contra el cual se dirigió el movimiento revolucionario, no están funcionando como tales normas, i.e., no están realizando las funciones regulativa y de criterio de la regularidad, lo que a su vez significa que el órgano en principio aplicador de dichas normas precedentes del orden evolucionado, las ha ignorado. Es perfectamente posible que si, en el caso, existiera la expansión del antecedente, los elementos que en dicha expansión se encontrarían serian distintos de los que aparecerían en el orden evolucionado contra el cual se dirigió el movimiento revolucionario. Ello permitiría comprender al movimiento revolucionario como un orden normativo previo al establecimiento del orden revolucionario triunfante $\mathrm{o}$, incluso, al fracasado.

En este caso, para el orden normativo en cuestión, han dejado de ser válidas las normas precedentes, en la medida en que no han determinado al acto normativo subsecuente $y$, por tanto, no han funcionado como normas. El sentido del orden normativo, que es determinar las condiciones de derrotabilidad de los enunciados condicionales que describen a las normas subsecuentes, ha sido rechazado y han aparecido uno o varios actos normativos que pueden adquirir validez en el caso de que existan actos normativos subsecuentes determinados por ellos. La longitud de la cadena normativa de un orden estable evolucionado se ha reducido, por la exclusión de los elementos correspondientes a las normas precedentes, lo que equivale a su desconocimiento. Éstas operan sobre las normas subsecuentes o determinadas como elementos que permiten su anulación, que es, como ya se ha mostrado, la verificación empírica de la verdad del enunciado condicional normativo que ha sido derrotado por la existencia de la negación de los elementos de la expansión de $p$. Por lo tanto, tenemos un corte en la cadena normativa primaria y evolucionada y la reducción en el número de los elementos de derrotabilidad de los enunciados condicionales que expresan el sentido del orden. Las nuevas normas, sin los elementos que las pueden anular, constituyen la instauración de un nuevo orden normativo, puesto que dichas normas tendrán que ser descritas con enunciados condicionales normativos inderrotables, instaurándose a partir de ellas un nuevo orden jurídico reducido. Esto puede acontecer en cualquier lugar de las diversas cadenas normativas posibles del orden primario evolucionado, generándose con ello una pluralidad de órdenes normativos con contenidos diversos, en ocasiones contradictorios y con ámbitos de validez espacial y personal variables. Por ello, la tesis que se sostiene es que, en una revolución, existen uno o varios órdenes normativos reducidos o revolucionarios en conflicto que se desarrollan y aplican, i.e., que son válidos, hasta que uno de ellos logra imponerse y establecer una constitución que será válida en el futuro, momento en el cual termina la validez de los órdenes revolucionarios. Generalmente el acto revolucionario original no sólo desconoce a las normas precedentes del orden primario, sino que esta- 
blece la norma fundamental positiva del orden revolucionario. La revolución francesa de 1789 tiene como acto fundacional revolucionario la decisión de la asamblea del tercer estado de sesionar en una sola asamblea con los representantes de los otros dos órdenes de la nobleza y el clero. Este acto revolucionario instauró a una autoridad suprema que generó una multiplicidad de normas pertenecientes al orden revolucionario. La revolución mexicana instauró la constitución del orden revolucionario con la proclamación del llamado Plan de Guadalupe hecha por Venustiano Carranza, plan que fue, incluso, el fundamento jurídico de la instalación del Congreso Constituyente de 1916-1917 que emitió la Constitución actualmente en vigor. Siempre se encuentra uno o varios órdenes normativos revolucionarios y su extinción a partir del momento en que uno de ellos se impone sobre los demás, estableciendo un árbol normativo con diversas cadenas normativas de longitud máxima, lo que significa que se instaura un orden jurídico que se individualiza y, por tanto, que es eficaz. Por tanto, toda revolución entraña el desconocimiento de ciertas normas y la existencia de otras que constituyen órdenes reducidos y parciales, que representan con los conceptos normativos la lucha revolucionaria, la que no es otra cosa que la ejecución de esos órdenes, su efectividad.

\section{CONCLUSIONES}

En consecuencia, en el caso de una revolución, con base en los conceptos apuntados, encontramos lo siguiente:

a) Un orden normativo primario evolucionado, contra el que se dirige el orden revolucionario y que constituye el parámetro para medir las conductas humanas que lo validan y reconocen o la invalidan y desconocen.

b) La característica central de este orden normativo primario es la existencia de una multiplicidad de cadenas normativas de longitud máxima, lo que significa, en la terminología tradicional, que es un conjunto de normas que en general es eficaz.

c) Una pluralidad de órdenes normativos, constituidos por cadenas normativas existentes reducidas, que poseen ámbitos de validez espacial y personal, de carácter variable, cuya ejecución es la lucha revolucionaria.

d) Estas cadenas normativas reducidas no permiten colocar en los enunciados condicionales normativos que las describen, los elementos correspondientes a las normas primeras de las cadenas normativas del orden primario evolucionado.

e) Nuestro modelo es compatible con el hecho, observable empíricamente, de que el contenido de los órdenes normativos reducidos se encuentra ideológicamente opuesto al contenido del orden primario evolucionado, en algunos aspectos fundamentales.

f) El conflicto que se da entre los órganos del orden primario evolucionado y los del orden u órdenes revolucionarios reducidos, es la expresión normativa de las contradicciones y oposiciones entre la pluralidad de ordenaciones válidas durante la lucha revolucionaria.

g) El orden primario evolucionado propende a conservar la longitud máxima de sus cadenas normativas y los órdenes normativos reducidos de la revolución a convertirse en un orden primario evolucionado.

b) Mientras coexisten los órdenes mencionados se da la guerra revolucionaria o el movimiento revolucionario. 
i) Cuando un orden revolucionario ha sustituido al orden primario evolucionado y se ha instaurado en su lugar, la revolución ha triunfado; si esto no acontece, ha fracasado y los órganos creadores del orden normativo reducido del orden revolucionario son generalmente sancionados como delincuentes por los órganos del orden primario evolucionado.

j) Mientras dura la guerra revolucionaria existen y son válidos dos o más órdenes normativos, cuya característica central es que sus ámbitos de validez personal o espacial son variables, dependiendo del éxito en los encuentros bélicos de los órdenes en conflicto.

Estas conclusiones exponen sumariamente las tesis que se han fundamentado en este escrito. Expresadas en otras palabras, diríamos que en toda revolución existe un orden revolucionario durante la lucha o guerra revolucionaria, el cual debe concebirse como una descentralización espacial o personal o ambas del orden estatal reconocido internacionalmente. Esto permite explicar la institución internacional del reconocimiento de beligerancia, y considerarlo provisionalmente como una guerra internacional.

\section{BIBLIOGRAFÍA}

AlCHOURRÓN, C., 2000: «Sobre Derecho y Lógica», en Isonomía. Revista de Teoría y Filosofía del Derecho, 13, octubre.

Alchourrón, C., y BulYgin, E., 1974: Introducción a la Metodología de las Ciencias Jurídicas y Sociales, Buenos Aires: Astrea.

- 1996: «Norma giuridica», en Analisi e Diritto, a cura P. COMANDUCCI y R. GUASTINI.

BAYÓN, J. C., 2000: «Derrotabilidad, Indeterminación del Derecho y Positivismo Jurídico», en Isonomía. Revista de Teoría y Filosofía del Derecho, 13, octubre.

GRICE, P., 1989: «Logic and Conversation», en Studies in the Way of Words, Harvard University Press.

HaRT, H. L. A., 1968: El Concepto del Derecho, 2. ${ }^{a}$ ed., Buenos Aires: Abeledo-Perrot, trad. G. R. CARRIÓ.

KelSen, H., 1934: Teoría General del Estado, Labor, trad. L. Legaz LaCAmBRA.

- 1946: La Teoría Pura del Derecho. Introducción a la Problemática Científica del Derecho, Buenos Aires: Losada, trad. J. G. TejerinA.

- 1949: Teoría General del Derecho y del Estado, México: Imprenta Universitaria, trad. E. GARCÍA MAYNez.

- 1979: Teoría Pura del Derecho, México: UNAM, trad. R. J. VeRnENGo.

- 1996: «¿Qué es un Acto Jurídico?», Isonomía. Revista de Teoría y Filosofía del Derecho, n. ${ }^{\circ} 4$, abril.

NAVARRO, P., 2000: «Sistemas Normativos, Derrotabilidad y Conocimiento del Derecho», en Isonomía. Revista de Teoría y Filosofía del Derecho, 13, octubre.

— 2000: «Derrotabilidad y Sistematización de Normas Jurídicas», en Isonomía. Revista de Teoría y Filosofía del Derecho, 13, octubre.

PRICE, J. J., 2001: Thucydides and Internal War, Cambridge University Press.

RodríGuez, J. L., y SuCAR, G., 1998: «Las Trampas de la Derrotabilidad. Niveles de Análisis de la Indeterminación del Derecho», en Analisi e Diritto. 
SCHmill, U., 1987a: «Reconstrucción Pragmática del Concepto del Deber Ser (Sollen)», en Teoría del Derecho y Conceptos Dogmáticos, UNAM.

- 1987b: «Introducción», a Problemas Capitales de la Teoría Jurídica del Estado, de H. KelSEN, México: Porrúa.

- 1996: «La Derogación y la Anulación como Modalidades del Ámbito Temporal de Validez de las Normas Jurídicas», Doxa, 19.

- 1997: Reconstrucción Pragmática de la Teoría del Derecho, México: Themis.

- 2000: «The Dynamic Order of Norms, Empowerment and Related Concepts», en Law and Philosophy, 19.

VON Wright, H., 1966: «Conditionality», en Six Essays in Philosophical Logic, p. 23, Acta Philosophica Fennica, vol. 60 (Helsinki: Academic Bookstore). 